\title{
Editorial
}

\section{Reading Physics Closely}

Nullius in verba, the sentence engraved on the Royal Society of London's coat of arms, famously captures the spirit of scientific inquiry. Often rendered as "Nothing in words," and more informally and correctly as "Don't take anybody's word for it," the motto counsels scientists not to rely on what others say, but to check it out for themselves. Looking and seeing, as opposed to relying on faith or authority, seems to express the way physics, like all science, works.

Yet physics is such a wordy discipline! Its practitioners and collaborators talk to each another with a mixture of common parlance and technical vocabulary. Reviewers criticize, teachers explain, advocates propound, grant applicants seek to convince, all with words. Furthermore, any and every attempt to articulate the meaning of experiments and theories, to oneself and others, uses words. The republic of physics thrives thanks to words. Its citizens never shut up.

Words do more than communicate and cultivate how physicists look and see; they also create the atmosphere in which physicists work, orienting why and where they look, shaping their understanding of what they perceive. According to Albert Einstein, if you want to understand the methods of theoretical physicists, "don't listen to their words, fix your attention on their deeds." But the essays in this issue revealing the opposite, showing how much those who seek to understand physics can learn from what physicists say. In her brilliant book Reading Popular Physics (2007), the Australian scholar Elizabeth Leane shows how close readings of the literature of popular physics yields important insights into culture. The articles in this issue engage in close readings in another area-the words of physicists- to achieve important insights into physics itself.

In the first article, for instance, Clayton Gearhart reveals new aspects of the work and results of James Franck and Gustav Hertz by reading their papers carefully. Using a little-known document-which he includes here in its first English translation-Gearhart puzzles through what these physicists thought their famous experiment meant, showing that their self-understanding changed significantly over time. The article argues that, though Franck and Hertz carried out their experiments during the time that Niels Bohr's now-famous atomic theory emerged, we cannot assume that they saw their work in terms of that theory.

In the second article, Dale Jaquette offers a close reading of Newton's texts to show the impossibility of fixing a firm boundary between physics and metaphysics, as Newton practiced both. Indeed, Newton found it especially hard to establish 
this boundary in his attempts to define space. As Jacquette explains it, the divine toy-maker may make the toys, but the problem is whether he did or did not also make space, the "toy-box" in which all things reside. In Newton's time, either alternative posed significant conceptual difficulties; Jacquette's close reading of Newton's texts highlights both these problems and Newton's less than satisfactory solution. The article asks us to consider the ways in which there is more to physics than the behavior of things alone.

Even flowers need reading, as we find in the final essay. John Nimmo, Paula Herman, M. B. Kirkham, and Edward R. Landa examine the studies that the physicist Lyman Briggs conducted concerning how mountain laurel flowers can fling their pollen, catapult-like, over considerable distances. They read Briggs's work in the larger context of his amazing career (including his role in founding the Manhattan Project), describing how he came to be concerned with the reproductive mechanisms of plants and how his physics background gave him sometimes more, sometimes less insight than other, more botanically trained, researchers.

We regard these papers as significant "experiments" in the craft of reading physics, and as indicating how indispensable words are in the practice of physics.

Which brings us back to nullius in verba, the Royal Society motto that seeks to capture the spirit of scientific inquiry: That motto is, after all, just words. How it could be otherwise? 